DOI: $10.5455 / 2320-1770$. ijrcog20130220

Case Report

\title{
An unusual case of postpartum convulsions
}

\author{
S. Bhanu Rekha* \\ Department of Obstetrics \& Gynecology, SVS Medical College \& Hospital, Mahabubnagar-509001, Andhra Pradesh, \\ India
}

Received: 7 January 2013

Accepted: 19 January 2013

*Correspondence:

Dr. S. Bhanu Rekha

E-mail: bhanurekha73@yahoo.com

\begin{abstract}
Convulsions in postpartum period are not very rare. Though the most common etiology is postpartum eclampsia, not all cases present with hypertension and proteinuria. These cases need to be reassessed with a CT or MRI to find out the other causes of convulsions like cerebral venous thrombosis, subarachnoid bleed, neurocysticercosis, tuberculomas etc. In our case, the patient presented with convulsions on the tenth postpartum day and her CT revealed hypo dense foci with perilesional edema in right high parietal region \& multiple hyper dense foci in bilateral cerebral hemispheres with no perilesional edema- old healed calcified granulomas. The patient was treated with phenytoin and albendazole.
\end{abstract}

Keywords: Convulsions, Neurocysticercosis

\section{INTRODUCTION}

Convulsions in the postpartum are not a rare presentation in obstetric emergencies. Most often the patient is treated as a routine case of eclampsia until she is investigated with other special investigations like CT or MRI when there is a suspicion as to evaluate the cause of convulsions in the absence of hypertension and proteinuria or when the patient does not respond to routine conventional anti-eclamptic regimes.

\section{CASE REPORT}

Mrs. X, aged 28 years came with history of an episode of convulsions on her 10th postpartum day. She was apparently alright before this. Patient had an episode of convulsions lasting for $2-3 \mathrm{mins}$ associated with headache and frothing of saliva. No h/o up rolling of eyes, tongue bites, involuntary urination/defecation, no h/o LOC or postictal confusion. There was no preceding history of blurred vision, epigastric pain, pedal edema, fever, cough, breathlessness or weakness of limbs. Patient gives h/o slight slurring of speech after the episode. She is P2L2, tenth post operated day, delivered by LSCS in both pregnancies, the indication for first LSCS being CPD, second indication being previous LSCS with CPD. She had regular antenatal checkups during both pregnancies and had no h/o pre-eclampsia or HTN in either of them and in between the pregnancies too. Ante partum, intra partum and postpartum periods of both the pregnancies were uneventful. Her past history was insignificant with no h/o any convulsions right from her birth. She consumes mixed diet but she is not a pork eater, sleep and appetite normal, bowel and bladder habits regular.

On examination, patient was drowsy and responding to verbal commands, no evidence of pallor, icterus, and lymphadenopathy. Afebrile and her vitals stable. Systemic examination was normal including the CNS examination. Fundoscopy was normal. On abdominal examination, uterus well involuted, no mass palpable. LSCS scar healed. On P/V examination lochia was healthy. $\mathrm{Hb} \% 12 \mathrm{gm} / \mathrm{dl}$, RBS $77 \mathrm{mg} / \mathrm{dl}$, Urine dipstick showed trace proteins, CUE was within normal limits, Renal and Liver function tests were normal. CT- hypo dense foci with perilesional edema in right high parietal region (nodular - granular stage of neurocysticercosis) and multiple hyper dense foci in bilateral cerebral hemispheres with no perilesional edema suggesting old healed calcified stage of neurocysticercosis (Figure 1). The stool examination provided no clue as to the presence of any proglottids or eggs. 


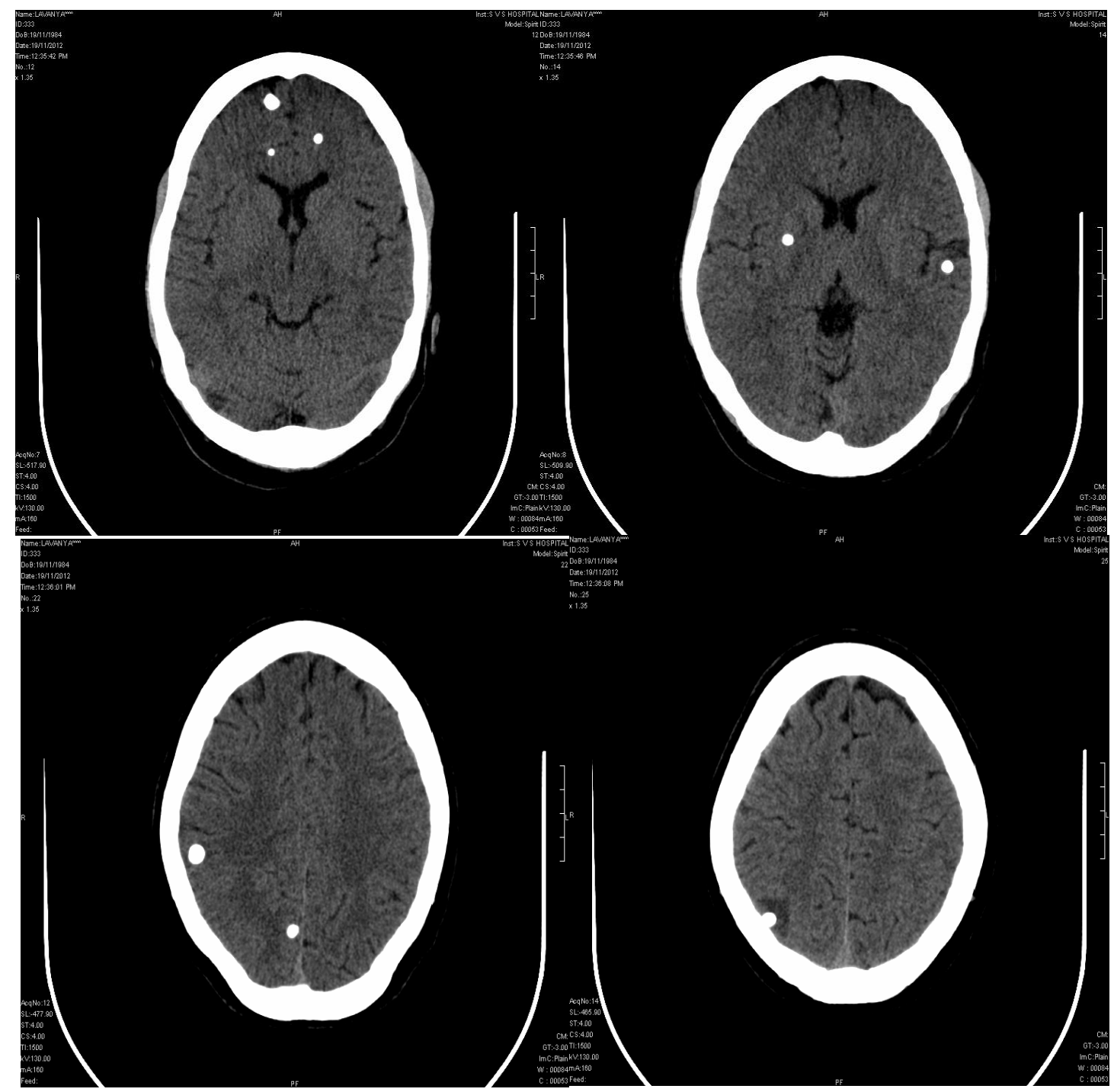

Figure 1: CT scan shows hypo dense foci with perilesional edema in right high parietal region and multiple hyper dense foci in bilateral cerebral hemispheres with no perilesional edema.

Serological tests were not done in her case to confirm the diagnosis. The patient was provisionally diagnosed to suffer neurocysticercosis. She was treated with an antiepileptic- Tab. Phenytoin 100mg 1+2, anti-cysticercal drug- Tab. Albendazole 400mg OD for 15 days, antiinflammatory- Tab. Prednisolone $5 \mathrm{mg}$ TID for 5 days, BD for 5days, OD for 5days. The patient was seizure free for 2 days and hence she was permitted to go home and review at OPD for further management.

\section{DISCUSSION}

Postpartum convulsions though not uncommon, can present because of varied reasons. The commonest diagnosis of postpartum convulsion being eclampsia, the other causes like cerebral venous thrombosis, cerebral hemorrhage, brain abscess, CNS tumors, viral encephalitis etc. Though a lesser common causes like neurocysticercosis, toxoplasmosis, tuberculomas, cerebral amebiasis should also be considered in our country which is still underdeveloped. In our case, the patient presented with convulsions on tenth postpartum day but she was normotensive and her urinary proteins was trace, a CT brain was ordered without any delay which helped us to diagnose the etiology as neurocysticercosis.

Taenia Solium infestation leads to cysticercosis in humans where the humans act as the intermediary host. The CNS involvement is not uncommon in these cases leading to neurocysticercosis. The classification of neurocysticercosis is parenchymal (92\% present with convulsions), subarachnoid, ventricular, spinal, muscular and disseminated.

Del Bruto et al have given diagnostic criteria for Neurocysticercosis. ${ }^{2}$ They are as follows: 
1. Absolute criteria

a. Histological proof

b. Fundoscopic evidence

c. Imaging (visualization of cysts)

2. Major criteria
a. Imaging suggestive of NCC
b. Immunological test
c. Plain $\mathrm{x}$ ray revealing soft tissue shadow

3. Minor criteria
a. Subcutaneous nodule
b. Intracranial calcification
c. Clinical manifestations
d. Response to cysticidal drug (lesions go away)

4. Epidemiological criteria
a. Patient belonging to an endemic area
b. Frequent travel to an endemic area
c. House-hold contact with Taenia Solium

Degrees of certainty can be classified into definitive, probable and possible by combination of one or more of above criteria.

\section{A. Definitive}

i. One absolute criteria

ii. Two major criteria

iii. One major + Two minor + One epidemiological criteria

B. Probable

i. One major + Two minor

ii. One major + One minor + One epidemiological criterion

iii. Three minor + One epidemiological criterion

C. Possible
i. One major criteria
ii. Two minor
iii. One minor + One epidemiologic

Whatever be the criteria, the commonest diagnostic tool is CT and MRI followed by serological tests- Enzyme Linked Immuno Electro transfer. Stool examination can rarely reveal proglottids and eggs. While in the nervous system, the T. Solium parasite goes through different stages of involution, which include the following ${ }^{3}$ :

- Vesicular stage: a viable parasite with a mild inflammatory reaction (CT- circumscribed \& hypo dense cysts).
- Colloidal stage: a parasite with a scolex in the process of degeneration and a severe inflammatory reaction around it (CT- ring enhancing lesions surrounded by perilesional edema).

- Granular stage: a parasite with a degenerated scolex and astrocytic gliosis around the cyst (CThomogeneously enhancing and calcified).

- Calcified stage: a parasite transformed into a calcified nodule with intense gliosis around the cyst (CT- hyper dense lesion).

The treatment includes an antiepileptic, anti cysticercal either albendazole $(15 \mathrm{mg} / \mathrm{kg} / \mathrm{day})$ or praziquantel for 28 30 days, corticosteroids- to ameliorate the inflammatory reaction of host to dying parasites. Surgery is rarely needed for cyst removal or for CSF shunts as in cases with hydrocephalus.

Prognosis is good with effective anti cysticercal drugs. Anti epileptics can be tapered in most cases. Prevention should be aimed at by taking effective measures like proper washing of vegetables and meat, adequate cooking, avoiding indiscriminate defecation and proper disposing of excreta, research for effective vaccination against T. Solium.

Funding: No funding sources

Competing interests: There are no competing interests to declare

Ethical approval: Not required

\section{REFERENCES}

1. Suarez VR, Iannucci TA. Neurocysticercosis in pregnancy: a case initially diagnosed as eclampsia. Obstet Gynecol 1999;93:816-8.

2. Ansari JA, Karki P, et al. Neurocysticercosis: a review article. Kathmandu Univ Med J (KUMJ) 2003;1:48-55.

3. Garg RK. Neurocysticercosis: a pictorial review. Infect Dis Clin Pract 2008;16:210-7.

DOI: $10.5455 / 2320-1770 . i j r \operatorname{cog} 20130220$

Cite this article as: Bhanu Rekha S. An unusual case of postpartum convulsions. Int J Reprod Contracept Obstet Gynecol 2013;2:101-3. 